\title{
BMJ Open Prospective cohort study of children with suspected SARS-CoV-2 infection presenting to paediatric emergency departments: a Paediatric Emergency Research Networks (PERN) Study Protocol
}

Anna L. Funk (D) , ${ }^{1}$ Todd A. Florin, ${ }^{2}$ Stuart R. Dalziel, ${ }^{3,4}$ Santiago Mintegi, ${ }^{5}$ Marina I. Salvadori, ${ }^{6}$ Daniel Joseph Tancredi (1) , ${ }^{7}$ Mark I. Neuman, ${ }^{8}$ Daniel C. Payne, ${ }^{9}$ Amy C. Plint, ${ }^{10,11}$ Terry P. Klassen, ${ }^{12}$ Richard Malley, ${ }^{13}$ Lilliam Ambroggio, ${ }^{14}$ Kelly Kim, ${ }^{1}$ Nathan Kuppermann, ${ }^{15}$ Stephen B. Freedman (D) ${ }^{16}$

To cite: Funk AL, Florin TA, Dalziel SR, et al. Prospective cohort study of children with suspected SARS-CoV-2 infection presenting to paediatric emergency departments: a Paediatric Emergency Research Networks (PERN) Study Protocol. BMJ Open 2021;11:e042121. doi:10.1136/ bmjopen-2020-042121

- Prepublication history and additional material for this paper is available online. To view these files, please visit the journal online (http://dx.doi.org/10. 1136/bmjopen-2020-042121).

ALF and TAF are joint first authors.

NK and SBF are joint senior authors.

Received 29 June 2020 Revised 09 November 2020 Accepted 01 December 2020

\section{Check for updates}

(C) Author(s) (or their employer(s)) 2021. Re-use permitted under CC BY-NC. No commercial re-use. See rights and permissions. Published by BMJ.

For numbered affiliations see end of article.

\section{Correspondence to} Dr Stephen B. Freedman; stephen.freedman@albertahealt hservices.ca

\section{ABSTRACT}

Introduction Relatively limited data are available regarding paediatric COVID-19. Although most children appear to have mild or asymptomatic infections, infants and those with comorbidities are at increased risk of experiencing more severe illness and requiring hospitalisation due to COVID-19. The recent but uncommon association of SARS-CoV-2 infection with development of a multisystem inflammatory syndrome has heightened the importance of understanding paediatric SARS-CoV-2 infection.

Methods and analysis The Paediatric Emergency Research Network-COVID-19 cohort study is a rapid, global, prospective cohort study enrolling 12500 children who are tested for acute SARS-CoV-2 infection. 47 emergency departments across 12 countries on four continents will participate. At enrolment, regardless of SARS-CoV-2 test results, all children will have the same information collected, including clinical, epidemiological, laboratory, imaging and outcome data. Interventions and outcome data will be collected for hospitalised children. For all children, follow-up at 14 and 90 days will collect information on further medical care received, and longterm sequelae, respectively. Statistical models will be designed to identify risk factors for infection and severe outcomes.

Ethics and dissemination Sites will seek ethical approval locally, and informed consent will be obtained. There is no direct risk or benefit of study participation. Weekly interim analysis will allow for real-time data sharing with regional, national, and international policy makers. Harmonisation and sharing of investigation materials with WHO, will contribute to synergising global efforts for the clinical characterisation of paediatric COVID-19. Our findings will enable the implementation of countermeasures to reduce viral transmission and severe COVID-19 outcomes in children.

Trial registration number NCT04330261

\section{Strengths and limitations of this study}

This multisite international research project has the unique ability to create a very large global dataset of children with COVID-19 and other infectious aetiologies that will include extensive details on exposures, symptoms, investigations, treatments and outcomes.

- This study builds on an existing multicentre research network to enable the rapid launch of a global study to address the COVID-19 pandemic.

By collecting data on both SARS-CoV-2-positive and -negative children, we will be able to conduct comparisons to develop models, using epidemiological and clinical characteristics, to predict paediatric SARS-CoV-2 infection.

- In the context of the quickly evolving COVID-19 pandemic, varying and evolving testing and control strategies are being put in place globally, which may limit ability to combine and generalise findings across different study sites.

- As testing at some sites may overwhelm research capacity, we will be unable to collect data on $100 \%$ of SARS-CoV-2 tested children.

\section{INTRODUCTION}

Paediatric COVID-19 following severe acute respiratory SARS-CoV-2 infection is not yet well characterised, and detailed reports on this topic are sparse. ${ }^{1-7}$ Reported infections are less common in persons younger than 18 years compared with older age groups. As of early June 2020, in reports with sample sizes of COVID-19 cases ranging from 4695 to 149082 , from China, the USA, Italy and Spain, individuals younger than 18 
years have constituted less than $2 \%$ of the total study cohort. $^{38-10}$

For reasons that remain elusive, children appear to be more likely than adults to present with asymptomatic or mild disease. ${ }^{11}$ The proportion of children with asymptomatic infections ranges between $4 \%$ and $16 \%{ }^{4}{ }^{12}$ However, these reports likely underestimate the frequency of asymptomatic carriage, as symptomatic patients are much more likely to be tested..$^{13}$ Moreover, symptoms in children appear to be less typical with only $73 \%$ of children with confirmed infection experiencing fever, cough or shortness of breath, which are reported in over $90 \%$ of infected adults. ${ }^{3}$ Even when symptomatic, children appear to be less likely to develop dyspnoea, hypoxaemia, acute respiratory distress syndrome and multiorgan system failure. ${ }^{12-14}$ Other symptoms, such as vomiting and diarrhoea, are present in 5\%-15\% of children $^{3}{ }^{4}$; in older children, a loss of the sense of smell (anosmia) has been noted as a possible predictor of infection. ${ }^{15}$ Furthermore, there is emerging evidence of a delayed multisystem inflammatory syndrome in children (MIS-C) that is linked to SARS-CoV-2 exposure that has been associated with severe outcomes. ${ }^{16-19}$

The reported proportion of children with COVID-19 requiring hospitalisation, although seemingly lower than that of adults, is variable and not well understood. In the USA and Canada, among children who tested positive for SARS-CoV-2, between $0.9 \%$ and $20 \%$ have reportedly required hospitalisation. ${ }^{30}$ Children with comorbidities and those aged $<1$ year appear to be at particularly high risk for hospitalisation, intensive care unit admission and death. ${ }^{3}{ }^{4}$ Although prior to mid-March, 2020, only two deaths had been reported among children worldwide, ${ }^{1}$ in a subsequent large case series of 2572 infected children in the USA, three additional deaths were reported. ${ }^{3}$

Though reports related to paediatric hospitalisation and mortality exist, there is a paucity of large-scale, multinational descriptions of the clinical features and disease course of children with COVID-19. Thus, a detailed examination of the risk factors for infection, clinical characteristics of infected children, predictors of severe outcomes and treatments provided, is urgently needed for this unique and potentially vulnerable population. As the symptoms of SARS-CoV-2 infection are a common reason for paediatric presentation to hospitals, the early identification of high-risk children and clinical phenotypes are vital to optimising care. Moreover, as viral shedding may occur in minimally symptomatic children, ${ }^{21}$ an in-depth study of paediatric infection characteristics, both mild and severe, will contribute to a better understanding of transmission risks. ${ }^{22}$ It will also be important to characterise and compare COVID-19 disease with other common paediatric respiratory illnesses to contextualise the severity of illness.

To achieve the aforementioned objectives, our study's primary aim is to fully evaluate clinical characteristics and outcomes of SARS-CoV-2 positive and SARS-CoV-2 negative children. Specifically, among children presenting to participating hospital emergency departments (EDs) with suspected infection, we will (1) describe and compare the clinical characteristics of SARS-CoV-2 positive children (ie, nucleic acid test-positive) and SARS-CoV-2 negative children (ie, nucleic acid test-negative) and (2) identify factors associated with severe outcomes in SARS-CoV-2 positive children (ie, intensive care unit admission, assisted ventilation, vasoactive medication use, death). The secondary aims of this study include (1) describing and comparing healthcare resource utilisation for patient management (eg, isolation, testing, imaging, supportive care) of both SARS-CoV-2 positive and SARS-CoV-2 negative children according to changes in national and regional policies and (2) describing the effects of changing case screening policies for the detection of SARS-CoV-2 in children.

\section{METHODS AND ANALYSIS \\ Study design and setting}

The Paediatric Emergency Research Network (PERN; https://pern-global.com/) represents the largest global paediatric acute care research collaboration, spanning 35 countries in four WHO regions representing $>200$ paediatric hospitals providing care to $>5$ million children annually. ${ }^{23}$ PERN represents a collaboration between seven existing paediatric emergency care medicine research networks-Paediatric Emergency Research Canada, ${ }^{24}{ }^{25}$ Paediatric Research in Emergency Departments International Collaborative, ${ }^{26}$ the Paediatric Emergency Medicine Collaborative Research Committee of the American Academy of Paediatrics, the Paediatric Emergency Care Applied Research Network, Research in European Paediatric Emergency Medicine (REPEM), Red de Investigacion y Desarollo de la Emergencia Pediatrica Latinoamerica and Red de Investigación de la Sociedad Española de Urgencias de Pediatría/Spanish Paediatric Emergency Research Group. ${ }^{27}$

In early 2019, the network launched the PERNPneumonia prospective cohort study to identify clinical predictors of severe outcomes in children diagnosed with community-acquired pneumonia. This global study includes 70 paediatric ED sites across 13 countries, including the USA, Canada, Spain, Italy, France, Switzerland, Romania, Argentina, Chile, Costa Rica, Paraguay, Australia, New Zealand and Singapore. The existence of the PERN-Pneumonia study has provided a unique opportunity to expeditiously initiate a prospective cohort study of children potentially infected by SARS-CoV-2. By building on the PERN-Pneumonia study's infrastructure (eg, ethics approvals, data-sharing agreements, electronic database, study teams), we were able to rapidly initiate the PERN-COVID-19 study to fill critical gaps in the understanding of COVID-19 in children.

PERN-COVID-19 is a prospective cohort study of children with suspected acute SARS-CoV-2 infections who present for care in one of 47 participating paediatric EDs located in 12 countries on four continents (see figure 1). 


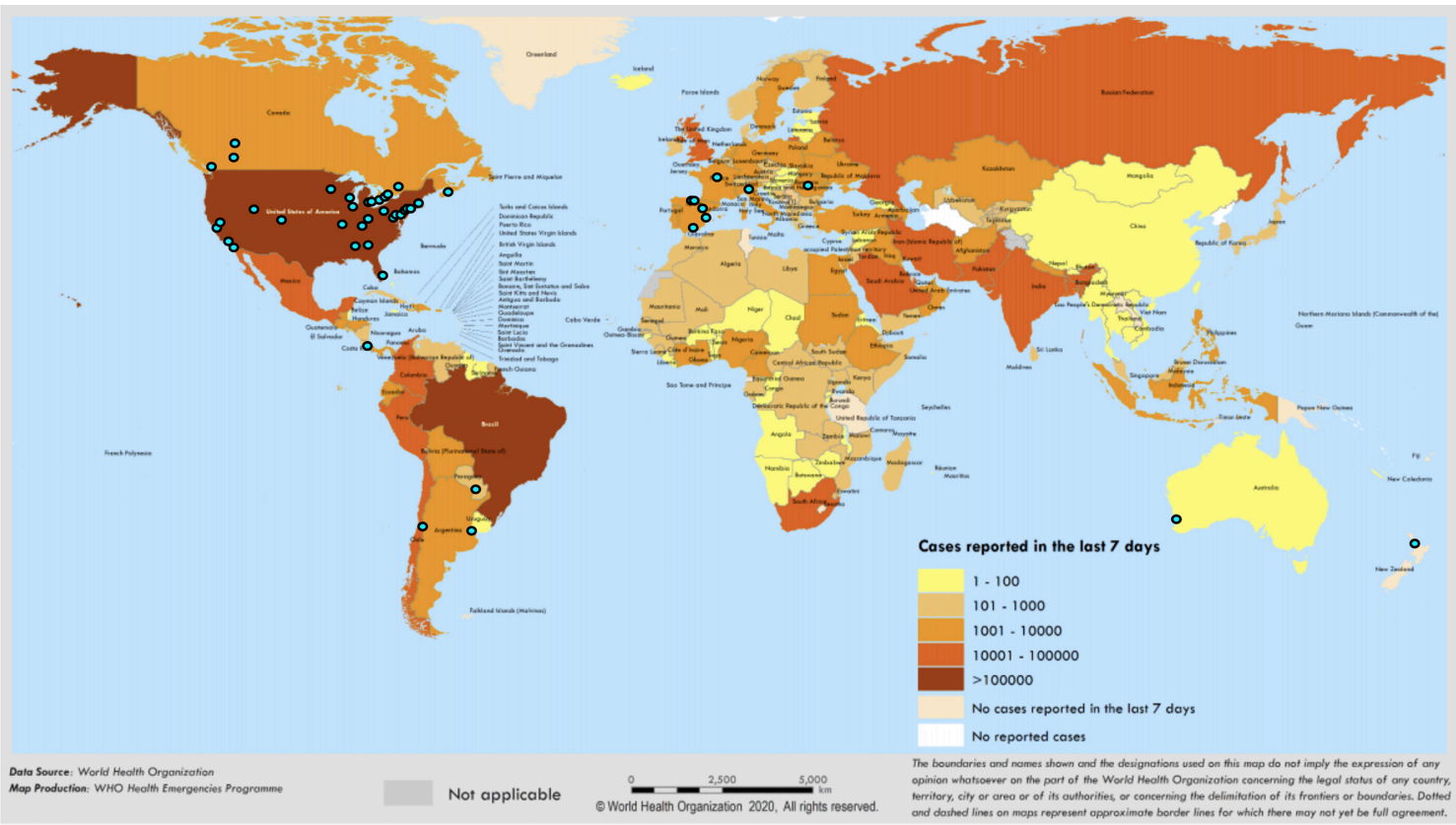

Figure 1 Locations of 47 participating PERN-COVID-19 study sites (blue dots) against a world map highlighting number of cases reported, by country, over the past 7 days (map adapted from reference 37. PERN, Paediatric Emergency Research Network.

Study recruitment began on 16 March 2020 at the Alberta Children's Hospital in Calgary, Canada. Most other participating sites initiated recruitment between mid-April and mid-May, 2020.

\section{Participants and recruitment}

Eligible participants are those younger than 18 years of age who present to a participating ED for care and who are tested for SARS-CoV-2 because of suspected acute infection (ie, symptoms, travel history or exposure history) according to local institutional policy. This recruitment strategy will identify children who are both SARS-CoV-2 positive and SARS-CoV-2 negative. The indications for testing children identified as potentially infected will differ by country, region and over time; however, standardisation of these protocols is beyond the control of this study. Participants are excluded if the caregivers and/ or children do not provide informed consent and assent, as required, respectively.

This pragmatic observational study does not specify the SARS-CoV-2 testing criteria, the specimen type collected for testing, or the SARS-CoV-2 nucleic acid test performed. In some sites, a combination of nucleic acid and serological tests may be performed. Information on all SARS-CoV-2 tests performed on study participant specimens will be collected. It is recognised that the standard procedures and criteria for SARS-CoV-2 testing are continuously evolving, often based on access to swabs, reagents and the regional epidemiology of disease. Therefore, detailed information on these policies will be reported weekly by sites. Local practices and illness severity will dictate decisions regarding isolation, investigations, treatments and hospitalisation.
Protocol implementation will vary by site to enhance feasibility. Sites may recruit participants in real-time as they present at the ED, or after the ED visit (eg, via a daily list of all children who presented to the ED and underwent SARS-CoV-2 testing). Contact procedures (eg, in-person or via telephone) and the consent process (eg, written or verbal) will adhere to each site's approved ethics agreements. In the instance of verbal consent attained via telephone, a detailed consent form is then emailed to the caregiver. All consents are recorded in the participant's case report forms (CRFs). Sensitive to the child's clinical condition and isolation policies, a research team member at each institution will contact the guardian to obtain informed consent and assent, as appropriate. The initial contact with hospitalised patients may occur in-person within the hospital or via telephone and will always occur by telephone if the patient has returned home (ie, not admitted or already discharged).

When the study was initiated, testing was limited at most sites and sites were instructed to attempt to recruit all children screened for SARS-CoV-2 infection. However, over time, the number of children screened increased, at which time the protocol was revised to have sites attempt to consecutively recruit the first five children tested each day. In sites located in areas where community prevalence of COVID-19 was very low, we moved to recruit the first two children tested each day. As enrolment may occur prior to SARS-CoV-2 test results becoming available, if none of the enrolled children on a given day are ultimately SARS-CoV-2 test-positive, then we will attempt to enrol up to two additional test-positive children. This is performed by returning to the list of children tested, and 


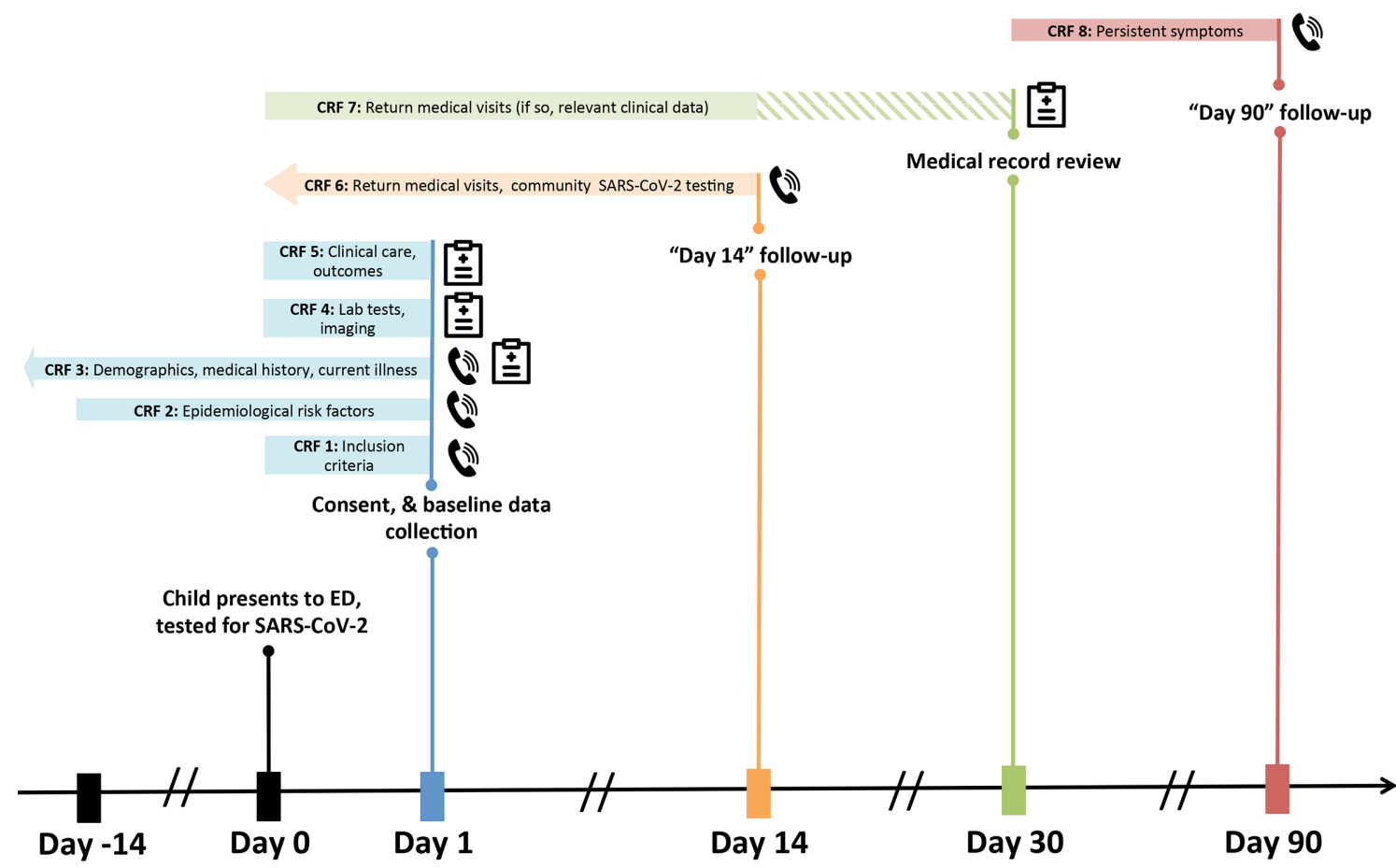

CRF = Case Report Form (2) = Telephone (or other method if $\begin{gathered}= \\ \text { preferred) interview with caregiver }\end{gathered}$

Figure 2 PERN-COVID-19 study participation timeline with data collection events. ED, emergency department; PERN, Paediatric Emergency Research Network.

continuing consecutively, attempting to recruit children consecutively with known positive test results.

\section{Data collection}

Participant demographic, epidemiological and clinical data will be collected at the time of enrolment in the ED, or shortly after. Clinical data will be collected throughout the period of hospitalisation (if relevant). At 14 and 90 days following the index ED visit, information on healthcare revisits and persistent symptoms will be collected, respectively (figure 2). Follow-up data collection time points were selected based on the presumed natural history of COVID-19 (ie, symptom progression 7-10 days after onset) while accounting for both short-term and longer-term potential complications. ${ }^{28}$ To complete case report forms (CRFs), study staff will consult hospital medical records and caregivers. A medical record review to verify 14-day outcome data will be performed which will also minimise lost to follow-up. We anticipate that children lost to follow-up without a hospital revisit did not experience an adverse outcome as study sites all serve as regional tertiary care centres, and thus, it is unlikely that a critically ill child would both be lost to follow-up and would be cared for elsewhere. Follow-up will be performed via telephone, text or email as per caregiver preference and as feasible based on-site resources and approvals. Our data forms are harmonised with the WHO recommended CRFs for clinical characterisation of COVID-19 (https://www.who.int/docs/default-source/ coronaviruse /who-ncov-crf.pdf?sfvrsn=84766e69_4). All research staff will be trained using a standardised and detailed manual of operations that includes explanations and examples for any potentially subjective questions in the study's data collection forms.

Baseline data: A study team member will complete the enrolment CRFs using the participants medical records and through a caregiver interview. Data collected will include: calendar date and site of enrolment, epidemiological risk factors (eg, recent travel, exposures), demographics (eg, age, sex), health information (eg, past medical history, vaccination status, comorbidities), onset of current illness (eg, timing, symptoms), imaging and laboratory investigations performed in the ED and treatments and care received (eg, intravenous fluids, antivirals, antibiotics).

Hospitalisation data: Enrolment CRFs will further capture management and outcome data related to events occurring during the initial hospitalisation, if required. Data collected will include length of hospital stay, level of hospital care required (eg, intensive care unit), laboratory tests, interventions and complications.

Fourteen-day follow-up: Day 14 follow-up will be conducted through telephone, text or email survey as per participant preference to determine if any additional healthcare visits or SARS-CoV-2 testing occurred. As appropriate, additional data related to medical care and treatments provided along with complications will 
be collected. If, at day 14 , a child has been continuously hospitalised since the time of initial ED presentation, this information will be captured.

Medical record review: To minimise missing data and supplement caregiver reports regarding additional medical care, a medical record review will occur 30 days after enrolment. This review will identify additional healthcare visits occurring up until 14 days following the initial ED visit. If the child was hospitalised during an eligible revisit, all outcome data available until the time of the review will be extracted.

Ninety-day follow-up: Day 90 follow-up will be conducted through telephone, text or email survey as per participant preference to determine if the participant is experiencing any ongoing symptoms that may be associated with the illness that prompted the initial ED presentation. Study team members will ask specifically about respiratory symptoms, psycho-behavioural concerns and other general, persistent, new or worsening health problems. If, at day 90, a child has been continuously hospitalised since the time of initial ED presentation, this information will be captured.

Site testing criteria and regional pandemic control measures: Every week, participating sites will complete a standardised log of their institution's testing policies (eg, symptoms and other risk factors prompting SARS-CoV-2 testing of children). In addition, they will record regional and national isolation and travel recommendations along with other pandemic control measures. Form completion will rely on institutional official documents, regional and national governmental websites and other sources, as required.

\section{Data storage}

A Research Electronic Data Capture (REDCap) database, that is, encrypted, password protected and de-identified, is serving as the master study database. Due to our desire to launch recruitment as quickly as possible, some study sites initiated data collection using paper CRFs and subsequently back-entered data into the REDCap database once it was made available.

\section{Sample size}

At the time of study development, there were many unknown factors related to the epidemiology of COVID-19 that precluded a robust sample size estimate. Our study design aims to enable the recruitment of more positive than negative cases relative to the prevalence in the general population, creating a cohort whereby $20 \%-40 \%$ of enrolled participants are confirmed to be SARS-CoV-2 positive. According to preliminary data from the USA the proportion of infected children with severe outcomes may be as high as $2 \% .^{3}$ Among children admitted or with a high likelihood of requiring hospitalisation who were tested for SARS-CoV-2 in Spain, approximately 10\% needed intensive care. ${ }^{9}$ In calculating a sample size we estimated that $2 \%$ of SARS-CoV-2 positive cases will experience severe outcomes. This is conservative estimate as the power of our study for assessing discriminative performance depends on the number of the less-frequent outcome level (ie, severe vs non-severe outcomes in SARS-CoV-2 positive children). For the severe outcomes, the predictive model will be limited to approximately 10 df. Recruiting 12500 SARS-CoV-2 screened participants ( 250 screened children, on average/site) to identify 50 severe outcomes in SARS-CoV-2 positive children using the most conservative assumptions (12 500×20\%x2\%) we will have $93.9 \%$ power to detect when the predictive model discriminating severe from non-severe outcomes truly (ie, in the larger population) has a c-statistic of 0.70 . These calculations used a variance inflation factor of 2.0 to account for model complexity (as measured by df) ${ }^{29}$ Power increases with higher assumed values for the c-statistic and with a higher number of assumed cases, attaining near certainty $(>99.9 \%)$ to detect $\mathrm{c}=0.70$ for the model of SARS-CoV-2 positive versus SARS-CoV-2 negative children (the other primary objective of this study). The principal investigators will analyse data and recruitment totals, alongside updated information from other studies on the behaviour of COVID-19, weekly, in order to determine the optimal data dissemination plans. Sample size calculations were performed using the SAS ROCPOWER macro. ${ }^{30}$

\section{Data analysis}

The following definitions will be used during data analysis:

- SARS-CoV-2 negative: Patient screened (ie, tested) but with a negative test result for SARS-CoV-2.

- SARS-CoV-2 positive: Patient screened (ie, tested) with laboratory confirmed SARS-CoV-2 infection.

- Severe outcomes: Positive pressure ventilation (invasive or noninvasive) or intensive care unit admission with ventilatory or inotropic support or $>48$ hours hospitalisation or severe diagnoses of heart, lung, kidney, vascular systems or death; other outcomes may be added as the understanding of the epidemic evolves.

- Ninety-day outcomes/persistent symptoms: a child will be considered as having a persistent symptom if the parents have indicated, at the 90-day follow-up, that respiratory, psychosocial or 'other' symptoms that began in the immediate time period surrounding the ED visit are persisting until the present day.

Statistical analysis: The collected epidemiological, demographic, clinical, laboratory and follow-up data for SARS-CoV-2 positive and SARS-CoV-2 negative participants will be summarised and compared using one-way analysis of variance, Kruskal-Wallis or Pearson $\chi^{2}$ tests, as appropriate. Bivariable analysis will be used to compare stratified (eg, by age category, region) severe outcome risk estimates among SARS-CoV-2 positive children to each other, as well as to similarly stratified severe outcome risk estimates among children who are SARS-CoV-2 negative. Conditional multiple logistic regression models, with matched groups based on hospital of enrolment, will be used to identify a set of independent variables that are 
able to: (1) discriminate among the two main case-statuses (ie, SARS-CoV-2 positive and SARS-CoV-2 negative) and (2) predict severe outcomes of COVID-19. For each of these two types of models, subgroup analyses using only a subset of the population of interest (eg, children younger than 90 days of age, children with chronic illnesses, etc) may be conducted. As there is a potential for misclassification with respect to infection status (eg, false negatives) due to the sensitivity and specificity of the SARS-CoV-2 tests used, sensitivity analyses using measurement error models and other strategies will be conducted. ${ }^{31}{ }^{32}$ The selection of variables for inclusion in the models will rely heavily on expert judgement among study investigators and will be supplemented by literature review. Strategies such as elastic-net regression will be used in order to improve the external generalisability of the model. Data on healthcare resource use across the two main case statuses will be compared using similar methods, with alternative specifications of link and distribution functions from the generalised linear model, as appropriate to account for outcome variable distributions. All data will be interpreted alongside changes to case screening and testing criteria and regional pandemic control measures. Our primary analyses will be complete case analyses, as we anticipate that key outcome and predictive variables will be available for a large fraction of the study population. However, sensitivity analyses evaluating the impacts of non-ignorable missingness on the soundness of our complete case inferences will be conducted and reported according to principled approaches to missing data. ${ }^{33} \mathrm{~A}$ detailed statistical analysis plan can be found in (online supplemental appendix).

\section{Patient and public involvement}

Patients and/or the public were not involved in developing the research questions or designing the study methods. This study currently has no plans to involve patients in the dissemination of study results. However results will be shared via social media and using knowledge translation tools designed to inform the public.

\section{Timeline}

As of 11 June 2020, most (31/47) study sites have obtained regulatory approvals and have initiated recruitment. We plan to continue recruitment for up to 12 months (ie, from April 2020 to March 2021), however, this will depend on the timing and intensity of the pandemic. Allowing for the completion of the 90-day follow-up period and 3 months to complete data analysis, we anticipate that all data will be available for dissemination within 18 months of study initiation.

\section{ETHICS AND DISSEMINATION}

The start-up, regulatory approvals, eligibility screening, recruitment, consent, data collection and data entry activities for this study arm will take place locally at each site. The study was first approved in Calgary (Canada) by the University of Calgary's Conjoint Health Research Ethics Board (REB18-0107). In the USA, similar to the PERN-Pneumonia study, sites have the option to rely on the Cincinnati Children's Hospital Medical Center Institutional Review Board (IRB) to act as the central IRB of record for this study. As this study is non-interventional, and collects no biological specimens, the risk to participants is minimal. There are no direct benefits or remuneration offered to children or families who participate in the study.

The greatest challenge to this study is the unpredictable nature of the COVID-19 pandemic and the public health policies that are enacted to control it. In the midst of this public health emergency, our study maximises feasibility and creates limited additional stress on healthcare systems and reduces the risk of transmission by minimising direct contact with infected patients. All CRFs can be completed by telephone, email or text messaged survey, and medical record review. As case-screening criteria will vary both by site and by time period, a limitation includes the potential for selection bias; our statistical modelling approaches are designed to account for some of this bias. There is also the potential for misclassification (eg, false negatives) within the SARS-CoV-2 positive and SARS-CoV-2 negative groups. In order to understand how the sensitivity of real-time polymerase chain reaction performed on nasopharyngeal and oropharyngeal swabs might lead to misclassification in our control group of SARS-CoV-2 negative children, we need to also consider the pretest probability of being infected. ${ }^{34-36}$ It is reasonable to believe that the pretest probability among children tested for SARS-CoV-2 due to suspected infection in EDs would still be relatively low at any one time, due to the highly sensitive screening criteria that are commonly used (eg, any child with fever). With a test sensitivity of $70 \%$ and a pretest probability of $15 \%$ (a conservatively high estimate) in children with any symptom potentially suggestive of SARS-CoV-2 presenting to participating EDs, the percentage of misclassification of the SARS-CoV-2 negative control group would be $5 \% .^{35}$ These limitations are addressed in our statistical analysis plan, which outlines the use of measurement error models to account for varying levels of misclassification in our study groups.

We will share the results of the data collected in real time as rapidly and as openly as possible. We will run descriptive interim analyses weekly to enable data sharing with key national and international stakeholders, our study partners and the public. The study team includes individuals representing several leading organisations including the Public Health Agency of Canada, the US Centers for Disease Control and Prevention, WHO and the United Nations Children's Fund (UNICEF). Through close communication and sharing of data with these stakeholder partners, study results may rapidly lead to evidence-based changes to, or confirmation of, institutional, regional, national, and international COVID-19 pandemic planning, case management policies and recommendations. As appropriate and relevant, our 
interim analysis outputs will be made available, via links, on our research network organisation websites. Findings will be shared in briefings and via social media platforms as appropriate. We will participate actively in relevant academic conferences and will publish all substantial findings in peer-reviewed, open-access journals. All articles will include citations to repositories that host the data underlying the results, together with details of any software used to process results. We will review and adapt our outputs management plan throughout the conduct of our project, as per discussion with all investigators as well as key knowledge users.

Harmonisation with the WHO recommended CRFs will enable us to rapidly share raw anonymous source data in keeping with the joint statement on sharing research data and findings relevant to the novel coronavirus $(\mathrm{nCoV})$ outbreak (https://wellcome.ac.uk/coronavirus-covid19/open-data). Anonymised data will be made available on reasonable request. Furthermore, we will collaborate and share the PERN-COVID-19 study protocol and CRFs openly with other study groups, such as the WHO Clinical Characterisation and Management Research Working Group, to support the synergising of global efforts to study paediatric COVID-19.

This study will assemble a substantial amount of data that will be analysed to identify predictors of severe COVID-19 in children, and risk factors for paediatric SARS-CoV-2 infection. These advances will allow for the earlier identification of confirmed SARS-CoV-2 infected children, assist with creating testing criteria and the prioritisation of those likely to have severe COVID-19. These advances have the potential to predict the presence of SARS-CoV-2 infection and severe outcomes in SARS-CoV-2 infected children. Healthcare resource utilisation will be optimised, which will improve resource availability, thereby optimising the care for those at greatest risk. Finally, this study will establish a global multicentre network poised to rapidly respond to novel infectious pathogens, which can urgently be reactivated for future public health crises.

\section{Author affiliations}

${ }^{1}$ Department of Pediatrics, Alberta Children's Hospital Research Institute, University of Calgary Cumming School of Medicine, Calgary, Alberta, Canada

${ }^{2}$ Division of Emergency Medicine, Ann and Robert H. Lurie Children's Hospital of Chicago, Northwestern University Feinberg School of Medicine, Chicago, Illinois, USA

${ }^{3}$ Departments of Surgery and Paediatrics: Child and Youth Health, The University of Auckland, Auckland, Auckland, New Zealand

${ }^{4}$ Children's Emergency Department, Starship Children's Health, Newmarket, Auckland, New Zealand

${ }^{5}$ Pediatric Emergency Department, Biocruces Bizkaia Health Research Institute, Hospital Universitario Cruces, Barakaldo, Spain

${ }^{6}$ Public Health Agency of Canada, Ottawa, Ontario, Canada

${ }^{7}$ Department of Pediatrics, University of California Davis, Davis, California, USA

${ }^{8}$ Division of Emergency Medicine, Boston Children's Hospital, Harvard Medical School, Boston, Massachusetts, USA

${ }^{9}$ National Center for Emerging Zoonotic Infectious Diseases, Division of Foodborne Waterborne and Environmental Diseases, Centers for Disease Control and Prevention, Atlanta, Georgia, USA

${ }^{10}$ Department of Pediatrics and Emergency Medicine, University of Ottawa, Ottawa, Ontario, Canada
${ }^{11}$ Division of Emergency Medicine, Children's Hospital of Eastern Ontario, Ottawa, Ontario, Canada

${ }^{12}$ Children's Hospital Research Institute of Manitoba, Department of Pediatrics,

University of Manitoba, Winnipeg, Manitoba, Canada

${ }^{13}$ Division of Infectious Disease, Boston Children's Hospital, Harvard Medical School, Boston, Massachusetts, USA

${ }^{14}$ Sections of Emergency Medicine, Department of Pediatrics, Children's Hospital Colorado, University of Colorado, Denver, Colorado, USA

${ }^{15}$ Departments of Emergency Medicine and Pediatrics, University of California Davis School of Medicine, Sacramento, California, USA

${ }^{16}$ Pediatrics, Cumming School of Medicine, Alberta Children's Hospital Research Institute, University of Calgary, Calgary, Alberta, Canada

Acknowledgements The PERN-COVID-19 Study team includes: Adam Isacoff (West Palm Beach, USA), Adriana Yock-Corrales (San José, Costa Rica), Alberto Arrighini (Brescia, Italy), Alexander Rogers (Ann Arbor, USA), Amit Kochar (North Adelaide, Australia), Ana Perez Aragon (Granada, Spain), Andrea Morrison (Milwaukee, USA), April Kam (Hamilton, Canada), Arjun Rao (Sydney, Australia), Ben Lawton (Meadowbrook, Australia), Bruce Wright (Edmonton, Alberta), Carlos Miguel Angelats (Valencia, Spain), Carmen Campos (Zaragoza, Spain), Claudia R. Morris (Atlanta, USA), Fahd Ahmad (St Louis, USA), Frederic Samson (Bilbao, Spain), Gerard Cheron (Paris, France), Ida Concha Murray (Santiago, Chile), Iker Gangoiti (Bilbao, Spain), Jonathan Cherry (Halifax, Canada), Joseph Zorc (Philadelphia, USA), Kajal Khanna (Palo Alto, USA), Kelly Bergmann (Minneapolis, USA), Kerry Caperell (Louisville, USA), Kristen Breslin (Washington D.C., USA), Maala Bhatt (Ottawa, Canada), Maren Lunoe (Pittsburgh, USA), Maria Kwok (New York City, USA), Maria Van Kerkhove (Geneva, Switzerland), Meredith L. Borland (Perth, Australia), Michelle Eckerle (Cincinnati, USA), Mihai Gafencu (Timisoara, Romania), Michael A Gardiner (San Diego, USA), Muhammad Waseem (New York City, USA), Naveen Poonai (London, Canada), Nicholas Watkins (Hobart, Australia), Nidhya Navanandan (Aurora, USA), Nipam Shah (Birmingham, USA), Nirupama Kannikeswaran (Detroit, USA), Oluwakemi Badaki (Baltimore, USA), Pedro Rino (Buenos Aires, Argentina), Pradip Chaudhari (Los Angeles, USA), Raoul Kamadjeu (Nairobi, Kenya), Sarah Becker (Salt Lake City, USA), Shane George (Southport, Australia), Shefali Jani (Sydney, Australia), Simon Craig (Clayton, Australia), Tristan Turner (Townsville, Australia), Usha Avva (Hackensack, USA), Vikram Sabhaney (Vancouver, Canada), Viviana Pavlicich (San Lorenzo, Paraguay), Yaron Finkelstein (Toronto, Canada).

Contributors SF, TF, NK, TK, SD and AF conceptualised the study and formulated the initial study protocol. SD, SM, MS, DJT, MN, DP, AP, TK, RM, LA and KK further developed and provided critical feedback on the study protocol. AF and SF drafted the manuscript. All authors edited and approved the final version of the manuscript.

Funding This work is supported by a Canadian Institutes of Health Research (CIHR) 2019 Novel Coronavirus (COVID-19) Rapid Research Funding Opportunity Clinical Management Operating Grant (Number: OV2-170706), an Alberta Children's Hospital Research Institute seed grant, as well as a University of Calgary-Alberta Health Services, Clinical Research Fund seed grant. ALF is supported by a University of Calgary Eyes High Postdoctoral Fellowship. SF is supported by the Alberta Children's Hospital Foundation Professorship in Child Health and Wellness. SD is supported by Cure Kids New Zealand. AP is supported by the University of Ottawa Research Chair in Pediatric Emergency Medicine.

Map disclaimer The depiction of boundaries on this map does not imply the expression of any opinion whatsoever on the part of BMJ (or any member of its group) concerning the legal status of any country, territory, jurisdiction or area or of its authorities. This map is provided without any warranty of any kind, either express or implied.

Competing interests None declared.

Patient consent for publication Not required.

Provenance and peer review Not commissioned; externally peer reviewed.

Supplemental material This content has been supplied by the author(s). It has not been vetted by BMJ Publishing Group Limited (BMJ) and may not have been peer-reviewed. Any opinions or recommendations discussed are solely those of the author(s) and are not endorsed by BMJ. BMJ disclaims all liability and responsibility arising from any reliance placed on the content. Where the content includes any translated material, BMJ does not warrant the accuracy and reliability of the translations (including but not limited to local regulations, clinical guidelines, terminology, drug names and drug dosages), and is not responsible for any error and/or omissions arising from translation and adaptation or otherwise. 
Open access This is an open access article distributed in accordance with the Creative Commons Attribution Non Commercial (CC BY-NC 4.0) license, which permits others to distribute, remix, adapt, build upon this work non-commercially, and license their derivative works on different terms, provided the original work is properly cited, appropriate credit is given, any changes made indicated, and the use is non-commercial. See: http://creativecommons.org/licenses/by-nc/4.0/.

\section{ORCID iDs}

Anna L. Funk http://orcid.org/0000-0003-2440-7553

Daniel Joseph Tancredi http://orcid.org/0000-0002-3884-7907

Stephen B. Freedman http://orcid.org/0000-0003-2319-6192

\section{REFERENCES}

1 Ludvigsson JF. Systematic review of COVID-19 in children shows milder cases and a better prognosis than adults. Acta Paediatr 2020;109:1088-95.

2 Parri N, Lenge M, Buonsenso D, et al. Children with Covid-19 in pediatric emergency departments in Italy. $N$ Engl $\mathrm{J} \mathrm{Med}$ 2020;383:187-90.

3 CDC COVID-19 Response Team. Coronavirus Disease 2019 in Children - United States, February 12-April 2, 2020. MMWR Morb Mortal Wkly Rep 2020;69:422-6.

4 Lu X, Zhang L, Du H, et al. SARS-CoV-2 infection in children. N Engl J Med 2020;382:1663-5.

5 Castagnoli R, Votto M, Licari A, et al. Severe acute respiratory syndrome coronavirus 2 (SARS-CoV-2) infection in children and adolescents: a systematic review. JAMA Pediatr 2020;174:882.

6 Mannheim J, Gretsch S, Layden JE, et al. Characteristics of hospitalized pediatric coronavirus disease 2019 cases in Chicago, Illinois, March-April 2020. J Pediatric Infect Dis Soc 2020;9:519-22.

7 Zachariah P, Johnson CL, Halabi KC, et al. Epidemiology, clinical features, and disease severity in patients with coronavirus disease 2019 (COVID-19) in a children's hospital in New York City, New York JAMA Pediatr 2020;174:e202430.

8 Wu Z, McGoogan JM. Characteristics of and Important Lessons From the Coronavirus Disease 2019 (COVID-19) Outbreak in China: Summary of a Report of 72314 Cases From the Chinese Center for Disease Control and Prevention. JAMA 2020;323:1239-42.

9 Tagarro A, Epalza C, Santos M, et al. Screening and severity of coronavirus disease 2019 (COVID-19) in children in Madrid, Spain. JAMA Pediatr 2020. doi:10.1001/jamapediatrics.2020.1346. [Epub ahead of print: 08 Apr 2020].

10 Livingston E, Bucher K. Coronavirus disease 2019 (COVID-19) in Italy. JAMA 2020;323:1335.

11 Castagnoli R, Votto M, Licari A, et al. Severe acute respiratory syndrome coronavirus 2 (SARS-CoV-2) infection in children and adolescents. JAMA Pediatr 2020;174. doi:10.1001/ jamapediatrics.2020.1467. [Epub ahead of print: 22 Apr 2020].

12 Dong Y, Mo X, Hu Y, et al. Epidemiology of COVID-19 among children in China. Pediatrics 2020;145:e20200702.

13 Cruz AT, Zeichner SL. COVID-19 in children: initial characterization of the pediatric disease. Pediatrics 2020;145:e20200834

14 Wang D, Hu B, Hu C, et al. Clinical characteristics of 138 hospitalized patients with 2019 novel coronavirus-infected pneumonia in Wuhan, China. JAMA 2020;323:1061.

15 Huang $L$, Zhang $X$, Zhang $X$, et al. Rapid asymptomatic transmission of COVID-19 during the incubation period demonstrating strong infectivity in a cluster of youngsters aged 16-23 years outside Wuhan and characteristics of young patients with COVID-19: a prospective contact-tracing study. J Infect 2020;80:e1-13.
16 Rivera-Figueroa El, Santos R, Simpson S, et al. Incomplete Kawasaki disease in a child with Covid-19. Indian Pediatr 2020;57:680-1. doi:10.1007/s13312-020-1900-0

17 Verdoni L, Mazza A, Gervasoni A, et al. An outbreak of severe Kawasaki-like disease at the Italian epicentre of the SARS-CoV-2 epidemic: an observational cohort study. Lancet 2020;395:1771-8. doi:10.1016/S0140-6736(20)31103-X

18 Viner RM, Whittaker E. Kawasaki-like disease: emerging complication during the COVID-19 pandemic. Lancet 2020;395:1741-3.

19 Riphagen S, Gomez X, Gonzalez-Martinez C, et al. Hyperinflammatory shock in children during COVID-19 pandemic. Lancet 2020;395:1607-8.

20 Government A. COVID-19 Alberta statistics: interactive aggregate data on COVID-19 cases in Alberta 2020. Available: https://www. alberta.ca/stats/covid-19-alberta-statistics.htm [Accessed 15 Jun 2020]

21 Hoehl S, Rabenau H, Berger A, et al. Evidence of SARS-CoV-2 infection in returning travelers from Wuhan, China. N Engl J Med 2020;382:1278-80.

22 Lipsitch M, Swerdlow DL, Finelli L. Defining the Epidemiology of Covid-19 - Studies Needed. N Engl J Med 2020;382:1194-6.

23 Klassen TP, Acworth J, Bialy L, et al. Pediatric emergency research networks: a global initiative in pediatric emergency medicine. Pediatr Emerg Care 2010;26:541-3

24 Bialy L, Plint A, Zemek R, et al. Pediatric emergency research Canada: origins and evolution. Pediatr Emerg Care 2018;34:138-44.

25 Plint AC, Johnson DW, Patel $\mathrm{H}$, et al. Epinephrine and dexamethasone in children with bronchiolitis. N Engl J Med 2009;360:2079-89.

26 Babl FE, Dalziel SR, Borland ML. Establishing a research network. J Paediatr Child Health 2020;56:857-63.

27 Mintegi S, Gómez B, de la Torre M. Progress of the Spanish Pediatric Emergency Research Group (RISEUP-SPERG). Emergencias 2015;27:208-9

28 Li AM, Chan CHY, Chan DFY. Long-Term sequelae of SARS in children. Paediatr Respir Rev 2004;5:296-9.

29 Hsieh FY, Lavori PW, Cohen HJ, et al. An overview of variance inflation factors for sample-size calculation. Eval Health Prof 2003;26:239-57.

30 Rc Z. A SAS $₫$ macro for estimating power for ROC curves OneSample and two-sample cases. Proceedings of the 20th SAS Users Group International Conference 1995:223.

31 Küchenhoff H, Mwalili SM, Lesaffre E. A general method for dealing with misclassification in regression: the misclassification SIMEX. Biometrics 2006;62:85-96.

32 JP B. Measurement Error: Models, Methods, and Applications (Chapman \& Hall/CRC Interdisciplinary Statistics. 1 ed. CRC Press, 2010.

33 Perkins NJ, Cole SR, Harel O, et al. Principled approaches to missing data in epidemiologic studies. Am J Epidemiol 2018;187:568-75.

34 Watson J, Whiting PF, Brush JE. Interpreting a covid-19 test result. BMJ 2020;369:m1808.

35 Woloshin S, Patel N, Kesselheim AS. False Negative Tests for SARS-CoV-2 Infection - Challenges and Implications. N Engl J Med 2020;383:e38.

36 Anand S, Montez-Rath M, Han J. Prevalence of SARS-CoV-2 antibodies in a large nationwide sample of patients on dialysis in the USA: a cross-sectional study. Lancet 2020.

37 Organization W-WH. Situation Report - 143 : Coronavirus disease 2019 (COVID-19) 2020. Available: https://www.who.int/docs/defaultsource/coronaviruse/situation-reports/20200611-covid-19-sitrep143.pdf?sfvrsn=2adbe568_4 [Accessed 12 Jun 2020]. 